\title{
Structure of solar convection: guesses and observational evidence
}

\author{
A. V. Getling \\ Institute of Nuclear Physics, Lomonosov Moscow State University, Moscow, 119992, Russia \\ email: A.Getling@ru.net
}

Abstract. Signs of quasi-regular structures in time-averaged photospheric images are described.

As Getling \& Brandt (2002a, b) reported previously, the procedure of time averaging applied to a 2-h interval of the 11-h La Palma series of granulation images (see Simon et al. 1994) had revealed signs of long-lived, quasi-regular photospheric structures "trenches" and "ridges" in the brightness relief, which form systems of concentric rings or parallel strips. These systems resemble some roll patterns known from laboratory experiments on Rayleigh-Bénard convection and may be an imprint of the pattern of subphotospheric convection.

Although that particular averaged image in which the structures were first detected still remains the best available one, constructing animations of running averages made it possible to identify trenching in the intensity relief in other averaged images. In addition to the La Palma series, we analysed the 45.5-h series obtained with the SOHO MDI instrument on 17-18 January 1997 (see Shine et al. 2000). This MDI series contains white-light images taken at a 1-min interval; they have a resolution of about $4.8^{\prime \prime}$. Both the subsonically filtered and unfiltered versions of the series were available.

Some attempts of algorithmic treatment of such averages were made. In particular, the best averaged image was analysed by A. A. Buchnev and V. P. Pyatkin (Institute of Computational Mathematics and Mathematical Geophysics, Novosibirsk, Russia) using the software package developed by them to detect linear and circular structures in aerospace images. Generally, the algorithm detects many spurious features, and the results are sensitive to the input parameters. Nevertheless, the angular distribution of directions of the detected linear elements is strongly anisotropic - these elements definitely "prefer" some particular orientations.

We constructed a special algorithm to analyse, for selected trial centres in the image, the radial distributions of the azimuthally averaged intensity (sequences of centres can be run by scanning given rectangular areas). It was found that some particular centres are characterized by especially large amplitudes of radial variation (see the centre labelled as 1 near the upper right corner and centre 0 in the upper left part of Fig. 1a) and by nearly periodic radial dependences (centre 0 ).

We emphasize that, in contrast to what Rast (2002) claims, averaged random fields with some parameters typical of granulation contain only isolated linear features rather than families of such features and do not resemble averaged solar images. The decline of contrast with the averaging time for averaged solar images is markedly slower than for averaged Rast's random fields (Brandt \& Getling 2004). An example of a highly trenched pattern is given in Fig. 1b, where numerous ridges and trenches forming rightward downward "hatching" can be seen. In this case, the anisotropy in the distribution of linear features is obvious (the averaging time is also $2 \mathrm{~h}$ ). In addition, two families of nearly 


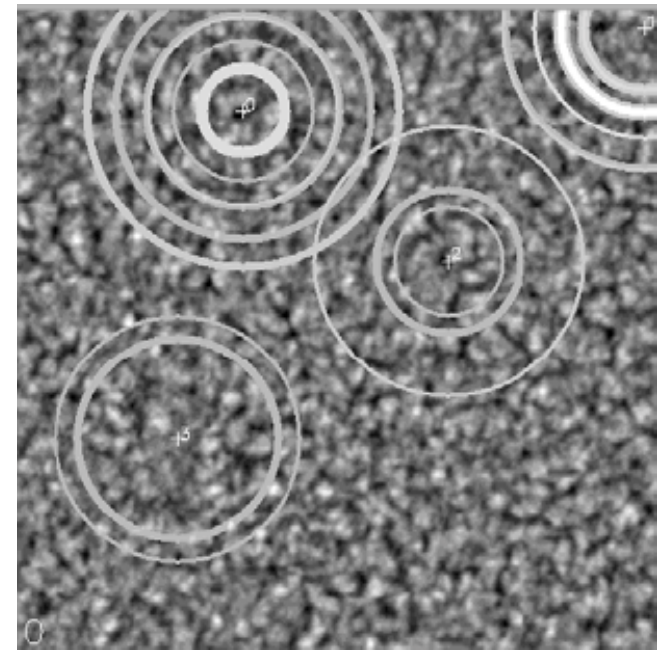

(a)

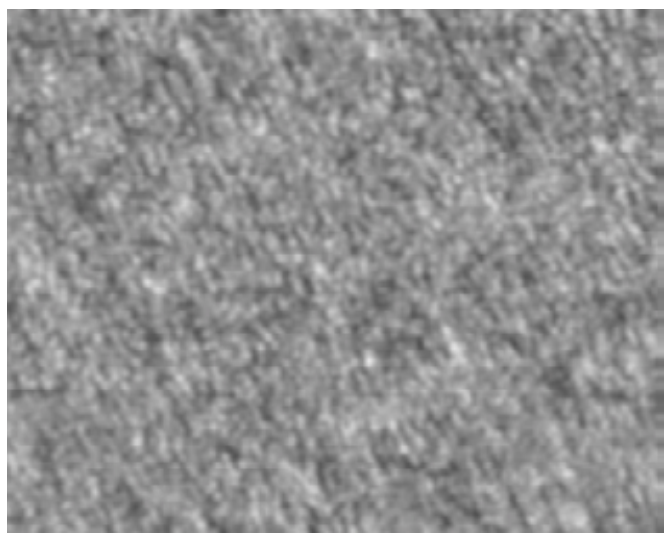

(b)

Figure 1. (a) Detection of ring patterns by the algorithm computing radial distributions of the azimuthally averaged intensity. All rings are plotted on a common scale of intensity. (b) Sample averaged image of the SOHO MDI series with a pronounced trenching pattern.

concentric, not quite regular rings can be distinguished. They are centred somewhere to the top right of the centre of the area shown and near the centre of its left half.

Families of concentric rings are sometimes superposed with radial "spokes", so that "web patterns" are observed. Ring and web patterns admit a hydrodynamic interpretation in terms of the development of certain instabilities of a larger-scale upwelling that can be associated with meso- or supergranules.

To conclude, we can state that patterns resembling straight- or circular-roll patterns of Rayleigh-Bénard convection do not seem unusual in the time-averaged granulation field.

\section{Acknowledgements}

I am grateful to R. A. Shine for the availability of the SOHO MDI data. This work was supported by the Deutsche Forschungsgemeinschaft and by the Russian Foundation for Basic Research (project code 04-02-16580-a).

\section{References}

Brandt, P. N. \& Getling, A. V. 2004 In Multi-Wavelength Investigations of Solar Activity, Proc. IAU Symp. No. 223. St Petersburg, 14-19 June 2004 (ed. A. V. Stepanov, E. E. Benevolenskaya \& A. G. Kosovichev), pp. ??-??. Cambridge University Press.

Getling, A. V. \& Brandt, P. N. 2002a Astron. Astrophys. 382, L5-L8.

Getling, A. V. \& Brandt, P. N. 2002b In Proc. 10th European Solar Physics Meeting, 'Solar Variability: From Core to Outer Frontiers'. Prague, Czech Republic, 9-14 September 2002. ESA SP-506, pp. 617-620.

Rast, M. P. 2002 Astron. Astrophys. 392, L13-L15.

Shine, R. A., Simon, G. W., \& Hurlburt, N. E. 2000 Solar Phys. 193, 313-331.

Simon, G. W., Brandt, P. N., November, L. J., Scharmer, G. B. \& Shine, R. A. 1994 In Solar Surface Magnetism, Proc. Workshop. Soesterberg, The Netherlands, 1-5 November 1993 (ed. R. J. Rutten \& C. J. Schrijver), pp. 261-270. Kluwer. 